\title{
Study for Performing Arts in 4th Industrial Revolution and Post-Corona Era:
}

\author{
Focusing on Zoom Generation
}

\author{
Na Hoon Park* \\ Department of Acting \& Performing Arts \\ Youngsan University \\ Yangsan City, South Korea \\ *bsmotel@hanmail.net
}

\begin{abstract}
The use of technology in the performing arts community facing the recent social disaster caused by the COVID-19 is increasing exponentially. This may be related to the influence of the 4th industrial era. The origin of the space for performing arts has been found in primitive society, civilized society, Greek period, and contemporary Proscenium and Black Box stages. The distribution of performances eventually through online and real-time live broadcasting is a contemporary issue that should be by addressed by the performing arts community, aside from the effect of COVID-19 crisis. This study, accordingly, examine the use of technology in contemporary performing arts community and the role of information $\&$ communication in the performing arts in the future by referring to a column by Nam Jeongsook, a culture planner, that emphasize the acquisition of information \& communication skill and publicity and another column by prof. Joh Eun-A that points out the limitation of online performance.
\end{abstract}

Keywords-information and communication, COVID-19, 4th industrial revolution, technology, Zoom

\section{INTRODUCTION}

The purpose of this study was to predict and discuss the performing arts paradigm in the post-corona era of the future, which is clearly meaningful given the recent phenomenon that the performance art is impossible without relying on information \& communication technology due to the pandemic of covid-19.

Walter Banjamin's theory on the value of Aura seems to have lost its significance in this technology replication era and the 4th industrial revolution era characterized by convergence, connection, decentralization, artificial intelligence, and internet. As artificial intelligence, a product of the 4th industrial era, appears as a way to replace creators in the performing arts community, a problem related to copyright arise [1], making the logic that we may not abandon the Aura of realism to be valid.

The ending declaration of Phantom of Opera, an influential musical, due to extended theater closure was an issue in performing arts community. Prior to this, Lloyd Webber, a producer of the Phantom of Opera, announced that "Show must go on" and provided his work to the audience of the world for free on the Internet. As shown above, the COVID-19 outbreak has unprecedentedly made all artists experience the production of creative works using media. Accordingly, the Zoom, a communication tool in the form of video conferencing, provided all the artists with a new way of creation [2]. Though it remains to be seen whether this phenomenon is simply a temporary phenomenon reflecting the current situation under the influence of COVID-19 or is a major phenomenon of the performing arts in the upcoming 4th industrial revolution era, it is natural to call the contemporary generation "Zoom Generation".

\section{TREnd \& CURRENT Status OF PERForming ARTS}

The site-specific performance is a recent new trend of performing arts that deviates from the theater performance paradigm and seeks to differentiate itself from the existing theater performing arts by addressing directly the city \& community and contemporary issues. The origin of the space for performing arts has been found in primitive society and it passed through the transition to civilized society, Greek period, and contemporary Proscenium and Black Box stages. Furthermore, street art and various types of performances in recent times, including performances in public spaces, and even in exhibition halls and museums, suggest that the performing arts creators are trying to meet audiences and learners in closer and deeper way and to communicate with them. It was also one of the processes of expanding the inner as well as the external scope of the performing arts. Erika Fischer's Aesthetics of Performativity, the epistemological basis for inducing active participation of the audience, realizes the audience participation through three principles: role reversal, community building, and contact [3]

The previous studies on site-specific performance, a type of performances in various spaces other than theaters, include White's study on the epistemological process in the 
performance origin theory and spatial form of modern performances [4], Radbourne's study on audience experience according to changes in performance space [5]. In addition, Ko studied, in the theater sociological perspective, the cases of site-specific performances in contemporary Korea [6].

These studies are significant examples that prove the change in the contemporary performing arts away from traditional theater-centered performance. They also may be an epistemological foundation to present the direction of performing arts in this era of COVID-19 crisis and 4th industrial revolution.

Then, how about the recent method of performances distribution using online live broadcasting and Zoom in this environment of performing arts that occupy streets and specific sites other than the theater? It remains to be seen whether the performing arts community accepts this form of performance based on the principle of Untact in online space without restrictions on physical distance.

As mentioned above relating to this question, it is necessary to compare the column by Nam Jeongsook, a culture planner, "The preparation by cultural art to deal with post-corona era" [7] and another column by Joh Eun-A, a professor of Kyunghee University, "It is difficult to immerse in online performance longer than 20 minutes" [8]. Nam, in her column, mentioned the job creation and achievement of publicity through the re-education of information \& communication technologies needed for performing artists in post-corona era, while Jo pointed out the fundamental limitations of such online performances. It is convincing that the value of Walter Benjamin's Aura mentioned above dominates the performing arts community that is based on immediacy and synchronicity, and this author, who is an artist, fully agrees to this.

Given, however, the recent crisis and the trend of contemporary performing arts where various changes are witnessed, it is questionable whether the traditional method for distributing and communicating performing arts will sustain the effectiveness. Furthermore, it may be claimed that the reeducation or capacity enhancement of artists through reeducation of information \& communication may help broaden the horizon of pure performing arts. This study, accordingly, presents the examples of Zoom performance that clearly showed the symbol of the current Zoom generation.

\section{CASES OF ZOOM PERFORMING ARTS}

"Thao and The Down Stay Down 'Phenom"” by Erin S Murray, a work of Zoom online video, clearly shows the liveliness in the productions by current Zoom generation. In this short video of about two minutes, they created interesting performance through appropriate use of zoom and angle editing. In Fig. 2 and 3, the compositional aesthetics of appearance and disappearance is performed to show various elements of choreography, and various forms of directional aesthetics that is hardly found in theater performances are shown in a special environment of Zoom. In Figure 3, particularly nine artists are creating one shape with only direction without physical contact.

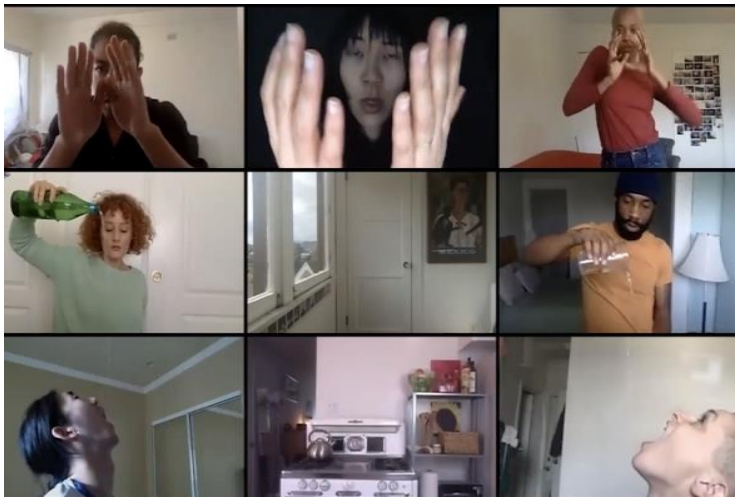

Fig. 1. Various composition and shape in space of screen.

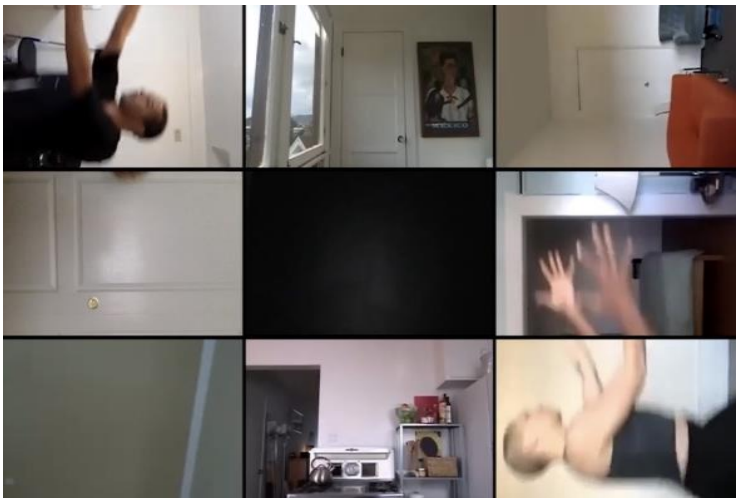

Fig. 2. Creation of new shape through screen design

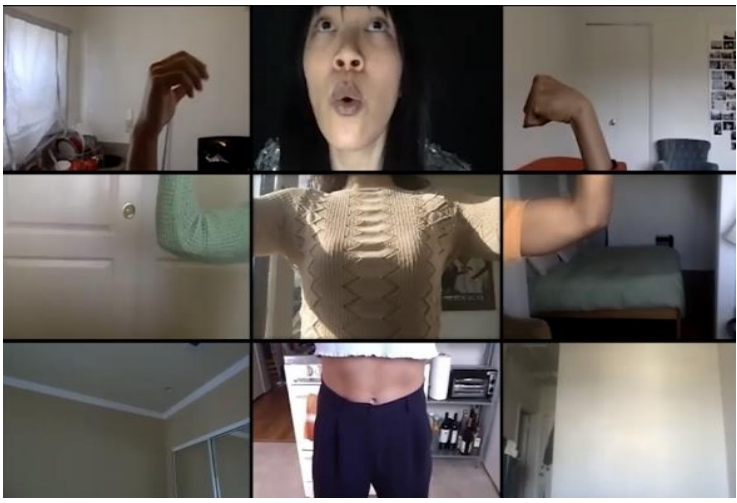

Fig. 3. Humorous scene

In addition, a humorous scene is produced by directing that uses the position of each artist on screen as the floor of a house and showing one in lower floor is drinking the water poured from the upper floor.

Phenom, the etymology meaning of which is marvelous person and genius, seems to create a way to induce communication between people in this cruel environment characterized by Untact. 


\section{CONCLUSION}

The work by Erin S Murray that was born due to the condition of this time such as COVID-19 crisis, development of internet, and 4th industrial revolution has sad background of creation so that it may not be regarded as a simple temporary creative work. Just as an opportunity found in crisis, this work may be a meaningful example of creation as a process to solve the challenges faced by performing arts community and present the vision for the upcoming 4th industrial revolution.

The change history of performing arts was briefly mentioned above while referring to the previous studies on sitespecific performance. The history of performing arts, from those in primitive places to those in various places based on the current paradigm of publicity, has made efforts to realize the unique values of art, that is, the role of a pioneer in reading contemporary value and presenting the future, according to the times and environment.

Aside from the COVID-19 crisis or the appearance of the 4th industrial era, art is likely to expand the area of art, while seeking constantly the aesthetic changes in the current area. As with the issue of copyright and the fundamental question about the scope of art, in relation to the role of artificial intelligence, are inextricably linked with the perspective of the endless vitality and possibility of art, the combination between the product of the 4th industrial revolution and the performing arts of the contemporary times should be accepted as a method to broaden the realm of art rather than being rejected because it undermines the uniqueness of art.

The limit of art, which is always open in this age of uncertainty, presupposes many uncertainties in itself. The aesthetics in dialectic of uncertainty should be reflected seriously.

\section{REFERENCES}

[1] K. Hristov, "Artificial intelligence and the copyright dilemma," IDEA, vol. 57 , pp. 431, 2016.

[2] Wikipedia, "Zoom (Software)" 2020. [Online]. Retrieved from: https://en.wikipedia.org/wiki/Zoom_(software)

[3] E. Fischer-Lichte, Ästhetik des performativen. Suhrkamp Verlag, 2012.

[4] G. White, Audience participation in theatre: Aesthetics of the invitation. Springer, 2013.

[5] J. Radbourne, H. Glow, and K. Johanson, The audience experience: A critical analysis of audiences in the performing arts. Intellect Books, 2013.

[6] Ko, An Analysis of Site-Specific Performance in Theater Sociological Perspective, Chung-Ang Graduate School, 2018, p 8.

[7] N. Jeongsook, "What will culture and art prepare for the post corona era?" $2020 . \quad$ [Online]. http://www.sctoday.co.kr/news/articleView.html?idxno=32607

[8] E. Joh, "Online performance immersion time, hard to exceed 20 minutes" $2020 . \quad$ [Online]. Retrieved http://m.kmib.co.kr/view.asp?arcid=0014613847 